\title{
Propiedades Volumétricas y Viscosimétricas de Soluciones Acuosas Diluidas de 1,4 Pentanodiol a varias Temperaturas
}

\author{
Manuel S. Páez ${ }^{(1)^{*}}$, Alfonso A. Portacio ${ }^{(2)}$, César Ortega ${ }^{(2)}$
}

(1) Universidad de Córdoba, Departamento de Química, Facultad de Ciencias Básicas,

Carrera 6 No. 76-103, Km 3, vía Cereté. Córdoba-Colombia (e-mail: mspaezm@unal.edu.co)

(2) Universidad de Córdoba, Departamento de Física, Facultad de Ciencias Básicas,

Carrera 6 No. 76-103, Km 3, vía Cereté. Córdoba-Colombia.

${ }^{*}$ Autor a quien debe dirigirse la correspondencia.

Recibido Feb. 26, 2010; Aceptado Abr. 13, 2010; Versión Final recibida Abr. 21, 2010

\section{Resumen}

Se determinó la densidad y la viscosidad de las soluciones acuosas diluidas de 1,4 Pentanodiol a temperaturas entre 283 y $308 \mathrm{~K}$, en un rango de concentración entre 0 y 0.006 en fracción molar. Se usó un picnómetro de cuello capilar tipo Wood-Brusie y un viscosímetro de Ubbelohde. El volumen molar parcial del soluto en función de la concentración de soluto presenta pendiente negativa para todas las temperaturas, lo cual indica que este soluto se comporta hidrofóbicamente. Los valores de viscosidad relativa fueron ajustados a una ecuación de segundo orden para obtener el coeficiente B que está relacionado con el tamaño y forma de la molécula del soluto.

\section{Volumetric and Viscometric Properties of Dilute Aqueous Solutions of 1,4 Pentanediol at various Temperatures}

\begin{abstract}
Density and viscosity of dilute aqueous solutions of 1.4 Pentanediol were determined at temperatures between 283 and $308 \mathrm{~K}$, in a concentration range between 0 and 0.0060 in mole fraction. A Wood -Brusie type capillary neck pycnometer and an Ubbelohde viscometer were used. The partial molar volume of solute in terms of the solute concentration showed a negative slope for all temperatures, indicating that solute behaves in a hydrophobic way. The relative viscosity values were adjusted to a second order equation to obtain the coefficient $B$, which is related to the size and shape of the molecule of solute.
\end{abstract}




\section{INTRODUCCIÓN}

Las propiedades termodinámicas y de transporte de soluciones acuosas juegan un papel muy importante en una variedad de campos: física, química, ingeniería química, proceso de separación, tratamiento de aguas residuales, eliminación de residuos químicos, control de contaminación, recuperación de petróleo, corrosión, entre otros. Sin embargo, el diseño y funcionamiento de procesos industriales que implican las soluciones acuosas requieren un conocimiento riguroso de los modelos y de datos experimentales de estas soluciones. (Mohammed, et al., 2009). El estudio de las interacciones no-covalentes (puentes de hidrógeno, interacciones electrostáticas, interacciones hidrofóbicas) proporciona información importante sobre la estabilidad conformacional y doblamiento/desdoblamiento del comportamiento de las proteínas globulares. (Banipal, et al., 2007) Dado que las proteínas son moléculas grandes y complejas, un estudio directo de las interacciones proteína-no electrolito es difícil. Por tanto se han desarrollado múltiples estudios (Romero, et al., 2002; Romero, et al., 2007; Bushuev, et al., 2008) sobre el comportamiento de las soluciones acuosas de solutos apolares y mixtos, para tratar de entender el efecto hidrofóbico, con lo cual los dioles y polioles en virtud de su carácter mixto se constituyen como candidatos naturales (modelos) que ayudarían a entender el efecto hidrofóbico de las interacciones de las biomoleculas en un ambiente acuoso.

\section{MATERIALES Y MÉTODOS}

Como reactivo se utilizó el 1,4 Pentanodiol del 99\% de pureza, Aldrich. El agua empleada para la calibración de equipos y preparación de soluciones fue doblemente destilada, con una conductividad menor que $2 \mu \mathrm{Scm}^{-1}$. Todas las soluciones fueron preparadas por el método de pesada usando una balanza OHAUS con una sensibilidad de $\pm 10^{-5} \mathrm{~g}$ en el rango más bajo. La densidad de las soluciones fue medida usando picnómetros de cuello capilar, tipo Wood-Brusie con una incertidumbre de $\pm 10^{-5} \mathrm{gcm}^{-3}$. La capacidad de estos picnómetros es de aproximadamente $80 \mathrm{~cm}^{3}$ los cuales fueron calibrados con agua a las temperaturas de trabajo. La viscosidad fue determinada usando un viscosímetro de Ubbelohde de nivel suspendido, con tiempos de flujo próximos a $300 \mathrm{~s}$ para el agua. La reproducibilidad de tiempos de flujo fue para todos los casos mejor que $0.05 \%$. Todas las medidas fueron realizadas en baños a temperatura constante y sus variaciones fueron monitoreadas con un termómetro digital Fluke Hart Scientific con una precisión de $\pm 0.001^{\circ} \mathrm{C}$.

\section{RESULTADOS Y DISCUSIÓN}

Los resultados experimentales de las densidades $(\rho)$ y las viscosidades $(\eta)$ de las soluciones acuosas de 1,4 Pentanodiol a $(283,15 ; 288,15 ; 293,15 ; 298,15 ; 303,15$ y 308,15$) \mathrm{K}$, en un rango de concentración entre (0.0000-0.0060) en fracción molar son reportadas en la tabla 1 , el volumen molar parcial del soluto se calcula a partir de la ecuación (1) (Romero, et al., 2007) y las tendencias para las soluciones acuosas diluidas de 1,4 Pentanodiol en función de la concentración se muestran en las figura 1 para las temperaturas en estudio. Estos datos están en concordancia en tendencias y en orden de magnitud con otros trabajos similares. (Kapadi, et al., 2001; George, et al., 2003)

$$
\bar{V}_{2}=V_{m}+x_{1}\left(\partial V_{m} / \partial x_{2}\right)_{T, P}
$$

Donde $V_{m}$ es el volumen molar, $\bar{V}_{2}$ es el volumen molar parcial del soluto, $x_{1}$ y $x_{2}$ son las fracciones molares del solvente y el soluto. La figura 1 , muestra que el volumen molar parcial del soluto en la región diluida aumenta linealmente con el aumento de la concentración de soluto para cada temperatura, esta correlación viene dada por la siguiente ecuación: 
$\bar{V}_{2}=x_{2}\left(\partial \bar{V}_{2} / \partial x_{2}\right)_{x_{2} \rightarrow 0}+\bar{V}_{2}^{\infty}$

Donde $\left(\partial \bar{V}_{2} / \partial x_{2}\right)_{x_{2} \rightarrow 0}$ y $\bar{V}_{2}^{\infty}$ son la pendiente limite y el volumen molar parcial a dilución infinita respectivamente, los resultados de estas cantidades para las soluciones acuosas diluidas de 1,4 Pentanodiol se muestran en la tabla 2 para cada temperatura.

Tabla 1: Densidades $(\rho)$ y viscosidades $(\eta)$ de las soluciones acuosas de 1,4 Pentanodiol a las temperaturas $(283.15 ; 288.15 ; 293.15 ; 298.15 ; 303.15$ y 308.15$) \mathrm{K}$

\begin{tabular}{ccccccc}
\hline $\mathrm{T} / \mathrm{K}$ & 283.15 & 288.15 & 293.15 & 298.15 & 303.15 & 308.15 \\
\hline $\mathrm{x}_{2}$ & \multicolumn{5}{c}{$\rho / \mathrm{g} \mathrm{cm}^{-3}$} \\
\hline 0.0000 & 0.99970 & 0.99910 & 0.99821 & 0.99705 & 0.99565 & 0.99403 \\
0.0020 & 0.99963 & 0.99904 & 0.99814 & 0.99699 & 0.99558 & 0.99397 \\
0.0025 & 0.99962 & 0.99902 & 0.99812 & 0.99699 & 0.99556 & 0.99396 \\
0.0030 & 0.99961 & 0.99902 & 0.99812 & 0.99699 & 0.99556 & 0.99396 \\
0.0035 & 0.99961 & 0.99902 & 0.99812 & 0.99700 & 0.99555 & 0.99397 \\
0.0040 & 0.99962 & 0.99903 & 0.99813 & 0.99701 & 0.99556 & 0.99398 \\
0.0045 & 0.99963 & 0.99905 & 0.99814 & 0.99703 & 0.99557 & 0.99399 \\
0.0050 & 0.99964 & 0.99906 & 0.99815 & 0.99705 & 0.99558 & 0.99401 \\
0.0055 & 0.99966 & 0.99908 & 0.99817 & 0.99708 & 0.99559 & 0.99403 \\
0.0060 & 0.99968 & 0.99911 & 0.99819 & 0.99710 & 0.99561 & 0.99404 \\
1.0000 & 0.99865 & 0.99554 & 0.99232 & 0.98905 & 0.98578 & 0.98248 \\
\hline $\mathrm{x}_{2}$ & & \multicolumn{5}{c}{$\mathrm{n} / \mathrm{mPa} \mathrm{s}$} \\
\hline 0.0000 & 1.304 & 1.138 & 1.002 & 0.893 & 0.797 & 0.709 \\
0.0020 & 1.369 & 1.193 & 1.050 & 0.926 & 0.829 & 0.739 \\
0.0025 & 1.385 & 1.205 & 1.060 & 0.938 & 0.839 & 0.747 \\
0.0030 & 1.403 & 1.223 & 1.074 & 0.947 & 0.848 & 0.756 \\
0.0035 & 1.421 & 1.236 & 1.085 & 0.957 & 0.857 & 0.765 \\
0.0040 & 1.438 & 1.251 & 1.097 & 0.967 & 0.865 & 0.775 \\
0.0045 & 1.455 & 1.264 & 1.107 & 0.977 & 0.874 & 0.782 \\
0.0050 & 1.473 & 1.278 & 1.120 & 0.987 & 0.881 & 0.791 \\
0.0055 & 1.493 & 1.295 & 1.133 & 0.997 & 0.889 & 0.799 \\
0.0060 & 1.516 & 1.311 & 1.145 & 1.009 & 0.898 & 0.807 \\
1.0000 & 275.471 & 186.880 & 132.579 & 95.602 & 69.662 & 51.891 \\
\hline
\end{tabular}

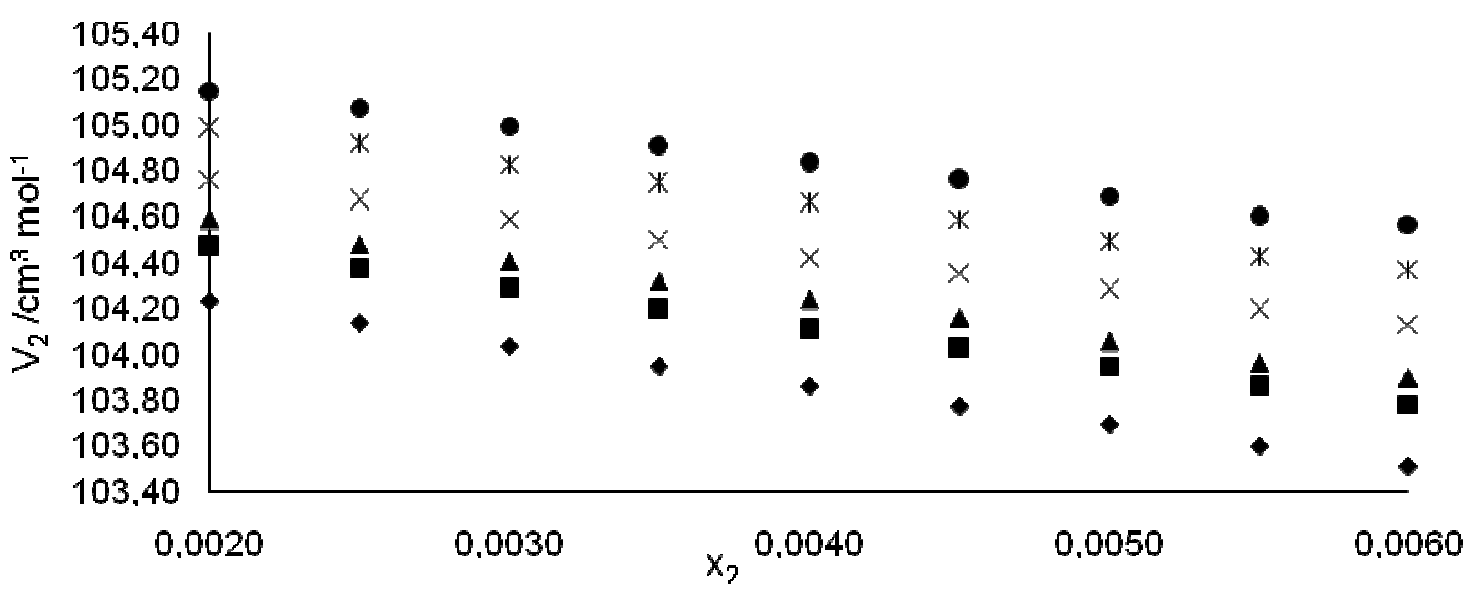

Fig. 1: Volumen molar parcial de las soluciones acuosas diluidas de 1,4 Pentanodiol en función de la concentración para las temperaturas: $(\triangleleft 283.15 ; \mathbf{n} 288.15 ; \boldsymbol{\Delta} 293.15 ; \mathbf{X}$ $298.15 ; * 303.15 ; \bullet 308,15) \mathrm{K}$ 
El análisis de los resultados de la tabla 2, indican el predominio de la interacción hidrofóbica sobre la hidrofílica y su concomitante disminución de este dominio con el aumento de la temperatura, en virtud a que la pendiente límite es negativa y aumenta en valor absoluto con el aumento de la temperatura.

Tabla 2: Pendientes limites, volumen molar parcia a dilución infinita, coeficientes $B$ y dB/dT para soluciones acuosas de 1,4 Pentanodiol a $(283.15 ; 288.15 ; 293.15 ; 298.15 ; 303.15$ y 308.15$) \mathrm{K}$

\begin{tabular}{ccccc}
\hline $\mathrm{T} / \mathrm{K}$ & $\left(\partial V_{2} / \partial x_{2}\right)_{x_{2} \rightarrow 0} / \mathrm{cm}^{3} \mathrm{~mol}^{-1}$ & $\bar{V}_{2}^{\infty} / \mathrm{cm}^{3} \mathrm{~mol}^{-1}$ & $\mathrm{~B} / \mathrm{Kg} \mathrm{mol}^{-1}$ & $\mathrm{~dB} / \mathrm{dT}$ \\
\hline 283.15 & $-188.0 \pm 0.4$ & $104.61 \pm 0.01$ & $0.54 \pm 0.02$ & -0.0177 \\
288.15 & $-182.0 \pm 0.3$ & $104.76 \pm 0.01$ & $0.49 \pm 0.02$ & -0.0167 \\
293.15 & $-172.0 \pm 0.3$ & $104.92 \pm 0.02$ & $0.46 \pm 0.01$ & -0.0157 \\
298.15 & $-170.0 \pm 0.2$ & $105.098 \pm 0.006$ & $0.43 \pm 0.01$ & -0.0147 \\
303.15 & $-164.0 \pm 0.6$ & $105.26 \pm 0.02$ & $0.40 \pm 0.01$ & -0.0137 \\
308.15 & $-118.0 \pm 0.9$ & $105.44 \pm 0.08$ & $0.38 \pm 0.01$ & -0.0127 \\
\hline
\end{tabular}

Tabla 3: Constantes de ajuste para la dependencia cuadrática del $\bar{V}_{2}^{\infty}$ con temperatura

\begin{tabular}{cccccc}
$\mathrm{C}_{1}$ & $\pm \Delta \mathrm{C}_{1}$ & $\mathrm{C}_{2}$ & $\pm \Delta \mathrm{C}_{2}$ & $\mathrm{C}_{3}$ & $\pm \Delta \mathrm{c}_{3}$ \\
0.00011 & 0.00004 & -0.033 & 0.002 & 105 & 3 \\
\hline
\end{tabular}

Por otra parte, es conocido que la segunda derivada del volumen molar parcial a dilución infinita con respecto a la temperatura, refleja el efecto de los solutos sobre la estructura del agua e indica el comportamiento hidrofóbico del soluto. El signo positivo se atribuye a que el soluto tiene un efecto formador sobre la estructura del agua y el signo negativo a que el soluto tiene un efecto disruptor. (Banipal et al., 2009) Siguiendo este orden de ideas un ajuste funcional del $\bar{V}_{2}^{\infty}$ con la temperatura mediante la ecuación cuadrática es presentado en la ecuación (3) y sus constantes de ajuste en la tabla 3; de acuerdo con la interpretación antes dada, podemos decir que en la región diluida el 1,4 Pentanodiol tiene un efecto formador sobre la estructura del agua, ratificando de esta forma lo obtenido con la pendiente límite.

$$
\bar{V}_{2}^{\infty}=c_{1} T^{2}+c_{2} T+c_{3}
$$

La tabla 2 también muestra el valor del coeficiente B inherente a la ecuación de Jones-Dole para soluciones de no electrolitos, tal como se indica a continuación. (Páez, et al., 2009)

$$
\eta_{r}=1+B m+D m^{2}
$$

Donde $\mathrm{m}$ es la molalidad, $\eta r$ es la viscosidad relativa, $B$ es un coeficiente que relaciona el tamaño y la forma del soluto en la molécula y los efectos de solvatación, mientras $D$ según (Páez, et al., 2009) representa un parámetro cuantitativo de interacción soluto-soluto y soluto-solvente a altas concentraciones. El significado usualmente atribuido al signo y la magnitud de $\mathrm{dB} / \mathrm{dT}$ refleja el efecto de los solutos sobre la estructura del agua e indica el comportamiento hidrofóbico del soluto. El signo negativo se atribuye a que el soluto tiene un efecto formador sobre la estructura del agua y el signo positivo a que el soluto tiene un efecto disruptor. En este trabajo no se analiza el coeficiente $D$, dado que la incertidumbre experimental en la determinación de este parámetro es mucho mayor que el parámetro mismo. Es evidente de los resultados presentados en la tabla 2, que este soluto se comporta hidrofóbicamente en la región infinitamente diluida y en consecuencia puede ser catalogado como un soluto formador de la estructura del agua, en esta región. La desviación de viscosidad fue calculada por la ecuación (5), donde $\eta_{m}, \eta_{1}, \eta_{2} x_{1}$ y $x_{2}$ son respectivamente la viscosidad de la mezcla, la viscosidad del agua la viscosidad del 1,4 Pentanodiol, la fracción molar del agua en la mezcla y la fracción molar del 1,4 Pentanodiol en la mezcla, en la figura 2, se correlacionan las desviaciones de viscosidad en función de la fracción molar para cada una de las temperaturas de trabajo. Adicionalmente valores negativos de la desviación de viscosidad pueden ser interpretados considerando las fuerzas de los enlaces hidrógeno intermolecular, el tamaño y la forma molecular de los componentes, de tal manera que grandes valores negativos de la desviación de viscosidad podrían indicar la formación de 
estructuras cíclicas de multímeros de especies diol-agua. Mientras que el efecto de la temperatura sobre una mezcla diol-agua puede ser analizado de la siguiente manera: si al aumentar la temperatura se produce una disminución del valor absoluto de la desviación de viscosidad entonces existe un debilitamiento de las interacciones diol-agua. (Portacio, et al., 2009; Kapadi, et al., 2001) Puede verse de los resultados de la figura 2, que las desviaciones de viscosidad de las mezclas acuosas de 1,4 Pentanodiol son todas negativas y sus valores disminuyen en valor absoluto con la temperatura, lo cual indica que las interacciones diol-agua se debilitan al aumentar la temperatura.

$\Delta \eta=\eta_{m}-\left(\eta_{1} x_{1}+\eta_{2} x_{2}\right)$

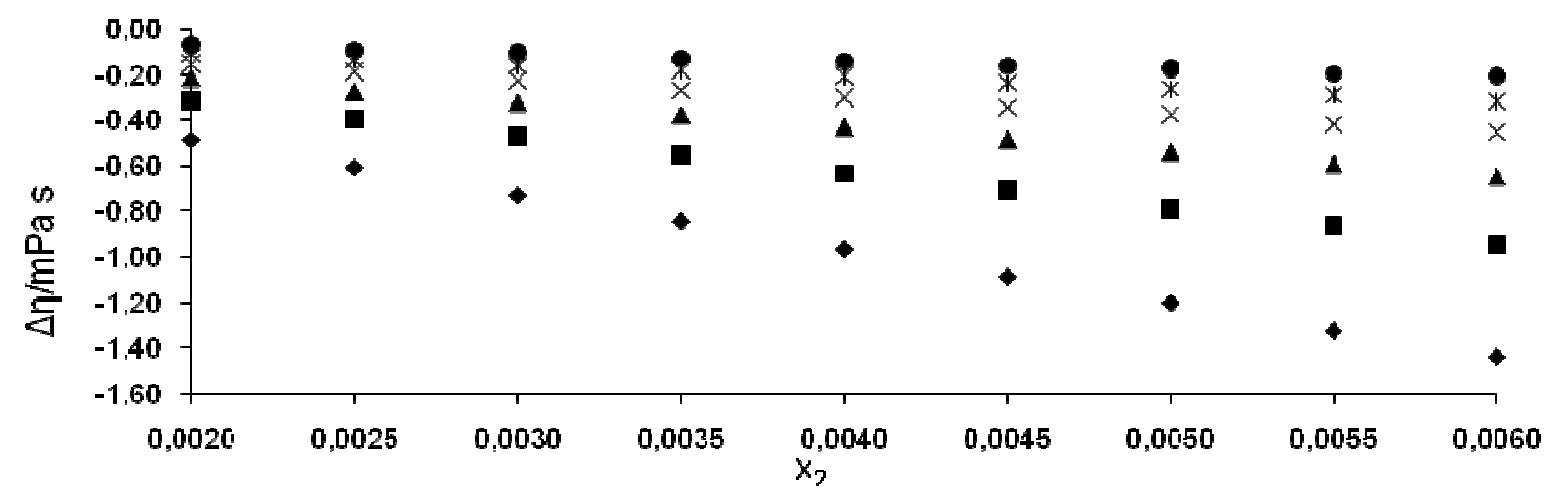

Fig. 2: Desviación de viscosidad de las soluciones acuosas diluidas de 1,4 Pentanodiol en función de la concentración para las temperaturas $(\bullet 283.15 ; \mathbf{m} 288.15 ; \boldsymbol{\Delta} 293.15 ; \mathbf{x} 298.15 ; * 303.15$;

- 308,15$) \mathrm{K}$

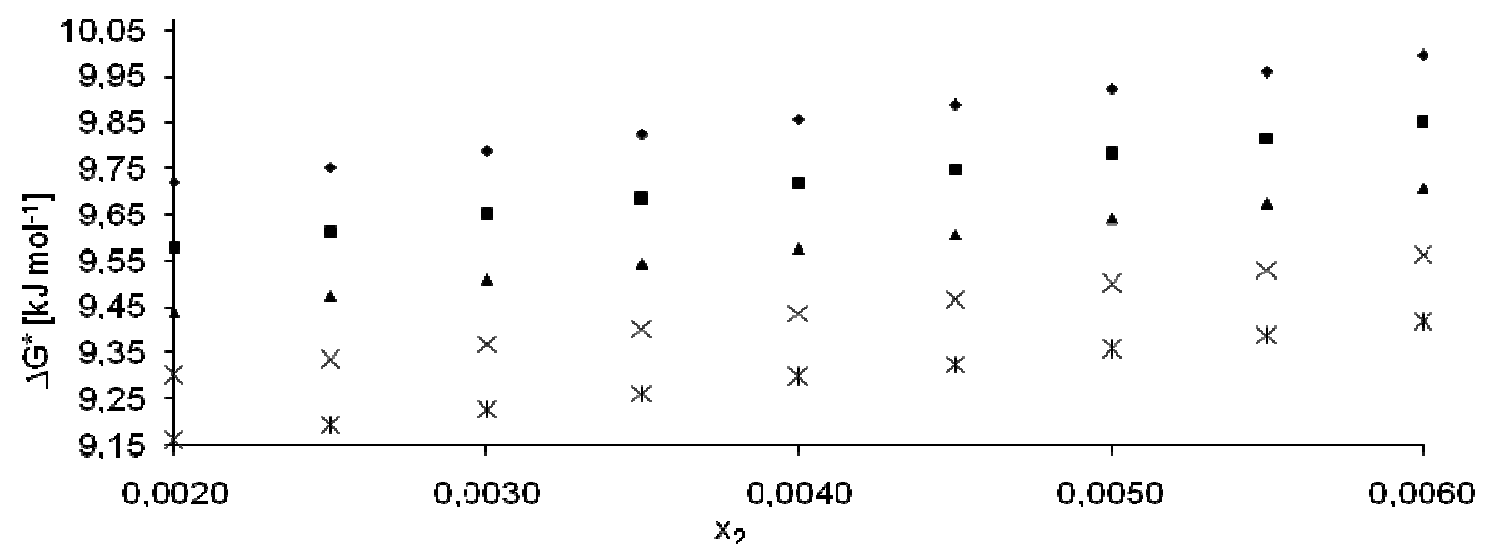

Fig. 3: Energía de Gibbs de activación para las soluciones acuosas diluidas de 1,4 Pentanodiol en función de la concentración a ( $283.15 ;-288.15 ; \boldsymbol{\Delta} 293.15 ; \mathbf{x} 298.15 ; * 303.15 ; \bullet 308,15) \mathrm{K}$

Finalmente, los parámetros termodinámicos de activación pueden ser evaluados aplicando la teoría de las velocidades absolutas de (Páez, et al., 2009), según la cual la viscosidad dinámica de un fluido Newtoniano está dada por la ecuación (6), donde $h$ es la constante de Planck, $N$ es número de Avogadro, $V_{m}$ es el volumen molar del líquido, $\Delta G^{\neq}$es la energía libre de Gibbs de activación para el proceso de flujo respectivamente y $\mathrm{T}$ es la temperatura absoluta. De acuerdo con esta teoría grandes valores positivos de $\Delta G^{\neq}$podrían asociarse con la formación de complejos a través de enlaces de hidrógeno intermoleculares entre moléculas similares y disimilares, los valores de $\Delta G^{\neq}$para las soluciones acuosas diluidas de 1,4 Pentanodiol en función de la concentración se muestran en las figuras se muestran en la figura 3 para las temperaturas de estudio y como puede notarse $\Delta G^{\neq}$presenta valores positivos para todas las concentraciones pero disminuyen con el aumento de la temperatura, indicando que las interacciones diol-agua se debilitan al aumentar la temperatura, lo que es consistente con los 
resultados obtenidos previamente., a partir de los resultados para la pendiente $\mathrm{dB} / \mathrm{dT}$ y de las desviaciones de viscosidad.

$\eta=\frac{h N}{V_{m}} \exp \left(\frac{\Delta G^{\neq}}{R T}\right)$

\section{CONCLUSIONES}

Se reportan datos experimentales para la densidad y la viscosidad absoluta en función de la concentración para región diluida $(0.0000-0.0060)$ en fracción molar a las temperaturas entre $(283.15$ - 308.15) K. También se reportan datos de volúmenes molares parciales a dilución infinita para el soluto a las temperaturas señaladas. Los resultados obtenidos para la segunda derivada del volumen molar parcial a dilución infinita con respecto a la temperatura, el coeficiente $c_{1}$ del ajuste funcional del volumen molar parcial del soluto a dilución infinita con la temperatura (ecuación 3), la derivada del coeficiente $B$ de la ecuación de Jones-Dole (ecuación 4) con respecto a la temperatura, indican que a bajas concentraciones predomina el fenómeno de hidratación hidrofóbica; en tanto que la desviación negativa de la viscosidad y los valores de la energía libre de Gibbs de activación, indican el favorecimiento de las interacciones hidrofóbicas del soluto con tendencias a la formación de complejos vía puentes de hidrogeno intermoleculares. También se detectó una concomitante destrucción de las interacciones hidrofóbicas con el aumento de la temperatura.

\section{REFERENCIAS}

Banipal, P., Chahal, A., Banipal, T; Volumetric Investigations on Interactions of Acidic/Basic Amino Acids with Sodium Acetate, Sodium Propionate and Sodium Butyrate in Aqueous Solutions. J. Chem. Thermodynamics: 36(11-12) 1635-1667(2007)

Banipal, P., Chahal, A., Banipal, T; Studies on volumetric properties of some saccharides in aqueous potassium chloride solutions over temperature range (288.15 to 318.15) K. J. Chem. Thermodynamics: $41(4)$ 452-483(2009)

Bushuev, Y., Davletbaeva, S., Korolev, V; Influence of the size of fixed-rigidity spheres on the structural and energy characteristics of hydrophobic hydration. Russian Chemical Bulletin, International Edition: 57 (9) 1811-1820 (2008).

George, J. y N. V. Sastry; Densities, Dynamic Viscosities, Speeds of Sound, and Relative Permittivities for Water + Alkanediols (Propane-1,2- and -1,3-diol and Butane-1,2-, -1,3-, -1,4-, and -2,3-Diol) at Different Temperatures. J. Chem. Eng. Data: 48(6), 1529-1539 (2003).

Kapadi, U.R., y otros cuatro autores; Studies of viscosity and excess molar volume of binary mixtures of propane-1,2 diol with water at various temperatures. Fluid Phase Equilibria: 192(1-2) 63-70 (2001).

Liu, H., Lin, R., Zhang, H; Enthalpic interactions of amino acids with imidazolein aqueous solutions at 298.15 K. Thermochimica Acta: 412 (1-2) 7-12 (2004).

Páez, M., Romero, C; Volumetric properties of aqueous binary mixtures of 1- butanol, butanediols, 1, 2, 4 butanetriol and butanetretol at 298.15K. Journal of Solution Chemistry: 36 (2) 237-245(2007)

Páez, M., Lafont, J., Portacio, A; Efecto de la temperatura sobre la viscosidad de soluciones acuosas diluidas de 1,2 Pentanodiol. Información tecnológica: 20 (2) 55-60(2009)

Portacio, A. y otros cuatro autores; Medidas de viscosidad de mezclas acuosas binarias a 293.15K. Revista Colombiana de física: 41 (2) 378-380(2009)

Romero, C., Páez, M; Thermodynamic Properties of aqueous alcohol and Polyol Solutions. Journal of Thermal Analysis and calorimetry: 70 (1) 263-267(2002)

Romero, C. y otros cuatro autores; Effect of temperatura on the volumetric properties of dilute aqueous solutions of 1,2 hexanediol. 1,5 - hexanediol; 1,6 - hexanediol and 2,5-hexanediol. Journal of Chemical Thermodynamics: 39 (8) 1101-1109 (2007) 